\title{
Low-amplitude variations detected by CoRoT in the B8Ille star HD 175869
}

J. Gutiérrez-Soto ${ }^{1,2,3}$, M. Floquet ${ }^{1}$, R. Samadi ${ }^{2}$, C. Neiner ${ }^{1}$, R. Garrido ${ }^{3}$, J. Fabregat ${ }^{4,1}$, Y. Frémat ${ }^{5,1}$, P. D. Diago ${ }^{4}$, A.-L. Huat ${ }^{1}$, B. Leroy ${ }^{2}$, M. Emilio ${ }^{6}$, A.-M. Hubert ${ }^{1}$, O. Thizy $^{7}$ L. Andrade ${ }^{8}$, B. de Batz ${ }^{1}$, E. Janot-Pacheco ${ }^{8}$, F. Espinosa Lara ${ }^{1}$, C. Martayan ${ }^{5,1}$, T. Semaan ${ }^{1}$, J. Suso ${ }^{4}$, M. Auvergne ${ }^{2}$, S. Chaintreuil ${ }^{2}$, E. Michel ${ }^{2}$, and C. Catala ${ }^{2}$

${ }^{1}$ GEPI, Observatoire de Paris, CNRS, Université Paris Diderot, place Jules Janssen, 92195 Meudon Cedex, France e-mail: juan.gutierrez-soto@uv.es

2 LESIA, Observatoire de Paris, CNRS, Université Paris Diderot, place Jules Janssen, 92195 Meudon Cedex, France

3 Instituto de Astrofísica de Andalucía (CSIC), Apartado 3004, 18080 Granada, Spain

${ }^{4}$ Observatori Astronòmic de la Universitat de València, Ed. Instituts d'Investigació, Polígon La Coma, 46980 Paterna, València, Spain

5 Royal Observatory of Belgium, 3 Avenue Circulaire, 1180 Brussels, Belgium

6 Departamento de Geociências, Universidade Estadual de Ponta Grossa, Av. General Carlos Cavalcanti, 4748 Ponta Grossa, 84.030-900, Brazil

7 Shelyak Instruments, Les Roussets, 38420 Revel, France

8 Universidade de São Paulo, Instituto de Astronomia, Geofísica e Ciências Atmosféricas - IAG, Departamento de Astronomia, Rua do Matão 1226, 05508-900 São Paulo, Brazil

Received 20 February 2009 / Accepted 13 July 2009

\section{ABSTRACT}

\begin{abstract}
Context. The origin of the short-term variability in Be stars remains a matter of controversy. Pulsations and rotational modulation are the components of the favored hypothesis.

Aims. We present our analysis of CoRoT data of the B8IIIe star HD 175869 observed during the first short run in the center direction (SRC1).

Methods. We review both the instrumental effects visible in the CoRoT light curve and the analysis methods used by the CoRoT Be team. We applied these methods to the CoRoT light curve of the star HD 175869. A search for line-profile variations in the spectroscopic data was also performed. We also searched for a magnetic field, by applying the LSD technique to spectropolarimetric data.

Results. The light curve exhibits low-amplitude variations of the order of $300 \mu$ mag with a double wave shape. A frequency within the range determined for the rotational frequency and 6 of its harmonics are detected. The main frequency and its first harmonic exhibit amplitude variations of a few days. Other significant frequencies of low-amplitude from 25 to a few $\mu$ mag are also found. The analysis of line profiles from ground-based spectroscopic data does not detect any variation. In addition, no Zeeman signature was found. Conclusions. Inhomogeneities caused by stellar activity in or just above the photosphere are proposed to produce the photometric variability detected by CoRoT in the Be star HD 175869. The hypothesis that non-radial pulsations are the origin of these variations cannot be excluded.
\end{abstract}

Key words. stars: emission-line, Be - stars: oscillations - stars: individual: HD 175869 - stars: rotation

\section{Introduction}

Be stars are non-supergiant B stars that show or have shown emission in Balmer lines, due to the presence of a decretion circumstellar disk. They rotate on average at $90 \%$ of the critical velocity (Frémat et al. 2005). Short-term variability is present in these stars, which is caused by non-radial pulsations or/and rotational modulation (see Porter \& Rivinius 2003).

In the HR diagram, late-type Be stars are located in the instability domain of slowly pulsating B stars (SPB) and therefore, high-order g-modes may be excited. Saio et al. (2007) identified the low-amplitude variations detected with the MOST satellite in the $\mathrm{B} 8 \mathrm{~V} \beta \mathrm{CMi}$ with prograde g-modes. They suggested that non-radial pulsations are involved in all rapidly rotating Be stars. Alternatively, Balona (1990, 1995) found a correlation between the photometric periods and their projected rotational velocities and proposed that the short-term variability in Be stars is caused by stellar spots or co-rotating clouds. For an updated description of Be stars, we refer to a paper by Neiner et al. (2009).

Since its launch in December 2006, the CoRoT satellite (Auvergne et al. 2009) has observed several Be stars in the seismology fields with a precision of several $\mu$ mag and during periods of time from 20 to 150 days.

HD 175869 (64 Ser) is a bright B8IIIe star $(V=5.56)$ observed in the CoRoT seismology field during the first short observing run of the center direction (SRC1, 27.3 days, from April 11 to May 8, 2007). This star was considered to be constant on the basis of Hipparcos data (Perryman et al. 1997). The high accuracy of the CoRoT light curve data, however, allows us to detect low-amplitude variations. The objective of this paper is to perform a complete and detailed analysis of the CoRoT light 
curve of this star and propose a preliminary interpretation of the origin of these variations. Detailed modeling will be published in a forthcoming paper.

We first describe the methods used by the CoRoT Be team to analyze the CoRoT data of the Be stars studied until now in the seismology fields (Sect. 2). In Sect. 3, we then report some instrumental features observed in the light curves. In Sect. 4, we present the analysis of the CoRoT light curve of the Be star HD 175869. Ground-based spectroscopic data was also acquired in order to complement the photometric analysis. The spectroscopic analysis is described in Sect. 5. Finally, we propose an interpretation related to the set of frequencies obtained in the analysis in Sect. 6.

\section{Methods for the frequency analysis}

Different methods have been used and developed by the CoRoT Be team to extract the maximum amount of information from the CoRoT light curve. Here we describe most of them and we highlight their differences and similarities.

The methods are divided in four types depending on their objectives: (i) search for frequencies; (ii) improvement of the frequency determination; (iii) determination of the amplitudes and phases for each frequency; and (iv) calculation of the significance of each frequency.

\subsection{Search for frequencies}

In the first stage, we search for frequencies in the light curves using methods based on the standard Fourier transform. These methods are pasper (Diago et al. 2008), Cleanest (Foster 1995), Clean-NG, and TiSAFT (Huat et al. 2008).

Pasper and Cleanest calculate the amplitude and the power spectrum for a wide range of frequencies, respectively, identify the frequency corresponding to the maximum peak in the Fourier space and then fit a sinusoidal function by means of least squares fitting to this frequency in the time domain. Pasper is based on the classical discrete Fourier transform (Deeming 1975), while Cleanest uses a fitting function based on a cosine function plus a constant (Foster 1995). The inclusion of a constant in the fitting is an advantage in relation to other Fourier methods because it is very convenient for data that are not equally dense, i.e., not regularly distributed in the time domain. The amplitude calculated with pasper is given in magnitudes, while the power spectrum computed with Cleanest corresponds to the $\chi^{2}$ distribution of the least squares fit. When a frequency is found, it is prewhitened by subtracting the corresponding synthetic sinusoidal light curve and a new search begins for the most powerful frequency in the residuals.

Clean-NG is a tool written by Leroy that uses Högbom's famous cleaning algorithm (Högbom 1974) to remove artifacts introduced by non-uniform sampling. Our implementation, which closely follows that of Roberts et al. (1987), has speed performances that have been tremendously improved by using modern methods developed for computing fast Fourier transforms of non-uniformly sampled data (Potts et al. 2001).

TiSAFT (Time Series Analysis with Fisher's Test) is a program written by Huat that searches for significant frequencies by fitting Lorentzian profiles in the Fourier domain. It iterates and stops when an identified frequency is not statistically significant according to the Fisher's test (Huat et al. 2008). This software also takes advantage of the non-uniform fast Fourier transform (Potts et al. 2001).

\subsection{Improve the frequency determination}

The second stage consists of improving with non-linear least squares fitting methods (local minimum) the estimation of initial frequencies detected with the methods described above. We employed the Period04 (Lenz \& Breger 2005), and two programs called Kurtz_bos and Improvef, developed by Gutiérrez-Soto and Emilio, respectively.

Period04 performs a non-linear least squares fitting to the data by using the Levenberg-Marquardt algorithm. Kurtz_bos is an automatic program that searches for frequencies using the efficient algorithm described in Kurtz (1985). Once a frequency has been detected, it scans a small range around this frequency to determine the minimum variance. The improved frequency is prewhitened from the data and another frequency is found in the residuals with the Kurtz algorithm. The code, then adjusts simultaneously the two frequencies allowing them to move to obtain the best fit. This method is iterated until a maximum number of frequencies is reached. Improvef uses the Powell algorithm (Powell 1964) to determine the set of frequencies that minimizes the fitting of a cosine function plus a constant to the time series.

\subsection{Determination of the amplitudes and phases}

To determine the amplitudes and phases of each detected frequency, we used a linear least squares fitting program called Amphi, written by Leroy, which is based on the SVD algorithm developed in the GSL library (Galassi et al. 2006). In this implementation, the matrix components are discarded if the ratio of singular values falls below a user-specified tolerance.

\subsection{Statistical significance of the frequencies}

A stop criterion is required to know whether the frequencies detected with these methods are significant or not, i.e., if the frequencies are related to noise or to signal. Pasper and Period04 use an empirical criterion based on the signal to noise ratio amplitude requirement described in Breger et al. (1993). Following these authors, a frequency is significant if the signal-to-noise ratio (SNR) for this frequency is higher than 4 . The TiSAFT code uses a statistical method to compute the significance of frequencies. For a given light curve and a given probability, TiSAFT calculates an amplitude threshold based on Fisher's test of significance in harmonic analysis (Fisher 1929).

\subsection{Error in the frequencies}

We calculated the frequency resolution following the Rayleigh criterion, i.e., $1 / T=0.03745 \mathrm{c} \mathrm{d}^{-1}$, where $T$ is the time duration of the light curve. However, Kallinger et al. (2008) propose a less conservative criterion, based on Monte-Carlo simulations, by using $1 /(4 T)$,

For the error in the frequency, amplitude, and phase values, we use the analytic formula from Montgomery \& O'Donoghue (1999). These errors are also calculated in the determination of amplitudes and phases by Amphi. The correlations in the residuals of the fitting (Schwarzenberg-Czerny 1991) are taken into account by multiplying the errors by $\sqrt{D}$, where $D$ is the number of consecutive datapoints that are correlated.

\section{CoRot light curves in the seismology fields}

We described some common properties of the light curves of the Be stars observed in the seismology fields of CoRoT. Our 


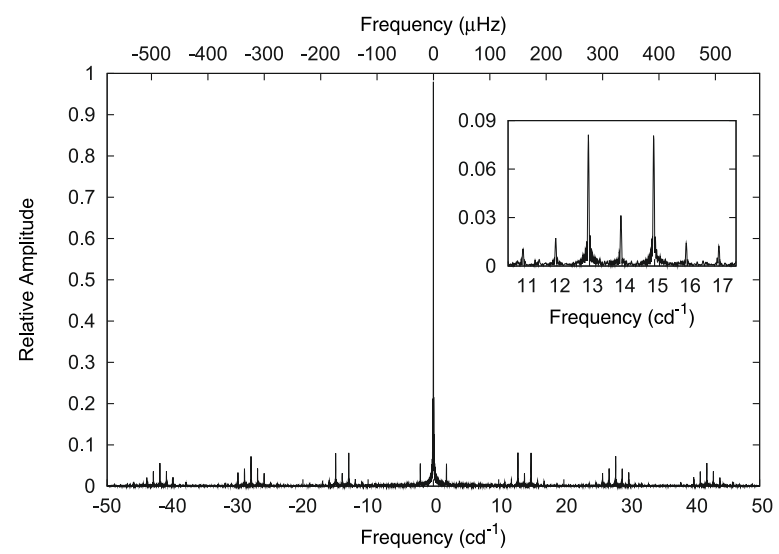

Fig. 1. Spectral window of the CoRoT light curve for the star HD 175869.

outline is based primarily on the CoRoT light curve of the star HD 175869, which lasts 27.3 days and has a data sampling of $32 \mathrm{~s}$. This star is representative of the majority of Be stars observed in the seismology fields of CoRoT until now, as explained below.

Two datasets are provided by the CoRoT pipeline: (i) the HEL dataset in which the dates are measured in the satellite reference frame and are transformed into the heliocentric reference frame, obtaining an irregular sampling; (ii) HELREG, where the dates are resampled onto a regular cadence in the heliocentric frame, with one measurement every $32 \mathrm{~s}$. We performed the frequency analysis in both datasets for the star HD 175869, obtaining similar results within the errors.

\subsection{Instrumental features}

We present some common instrumental features detected in the light curve of the Be stars observed in the seismology fields of CoRoT until now. The signal acquired by the CoRoT CCDs represented the convolution of the signal coming from the star with both the spectral window and the modulation of the orbit.

\subsubsection{Spectral windows}

Because of the passage of the satellite through the South Atlantic anomaly and to other low earth orbit perturbations (see Auvergne et al. 2009, for details), there are small gaps in the CoRoT light curve in phase with the orbital period. The percentage of lost datapoints is only $10 \%$ for HD 175869 . These gaps in the light curve produce a spectral window with aliases at the orbital frequency $\left(6184 \mathrm{~s}, 13.97 \mathrm{c} \mathrm{d}^{-1}, 161.7 \mu \mathrm{Hz}\right)$ and its harmonics, at the frequency related to the day/night variations $\left(2 \mathrm{c} \mathrm{d}^{-1}, 23.15 \mu \mathrm{Hz}\right.$ ) and combinations of them. In Fig. 1, we show the spectral window for the star HD 175869, and a zoom of the region about the orbital frequency.

In the majority of Be stars observed with CoRoT until now, the periodic signal originating in the star is within the frequency range of between 0 and $5 \mathrm{c} \mathrm{d}^{-1}(0-60 \mu \mathrm{Hz})$. Therefore, the CoRoT spectral window produces a signal in the same domain of the periodogram for all these stars. As shown in Fig. 2, aliases appear at frequencies $v_{\mathrm{star}} \pm n * 13.97 \pm 1 \pm 2 \mathrm{c} \mathrm{d}^{-1}$, where $n$ is a positive integer number, and $v_{\text {star }}$ is the stellar frequency. Therefore, for the majority of Be stars, aliases appear at frequencies higher than $10 \mathrm{c} \mathrm{d}^{-1}$, in groups of frequencies around the orbital frequency and its harmonics. Only aliases related to

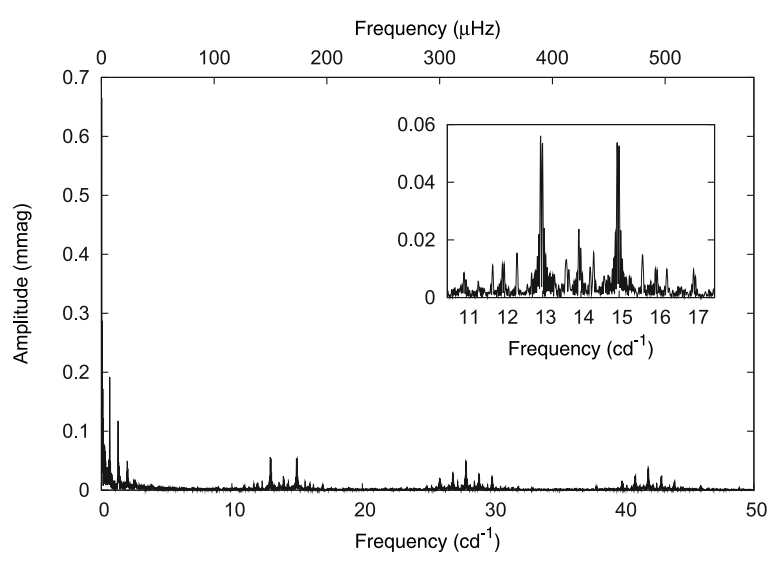

Fig. 2. Periodogram of the CoRoT light curve for the star HD 175869.

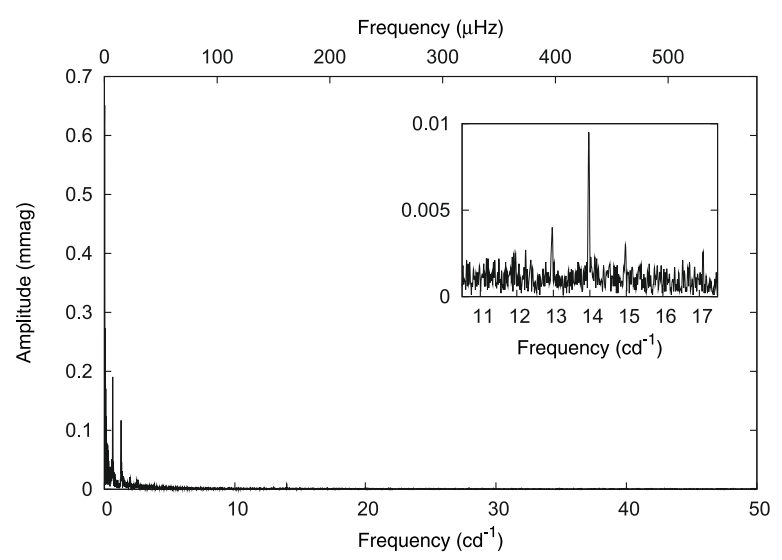

Fig. 3. Periodogram of the CoRoT light curve for the star HD 175869 , after filling the gaps with a linear interpolation.

day/night variations are in the range from 0 to $10 \mathrm{c} \mathrm{d}^{-1}$, but those have quite negligible amplitudes. We note that these aliases are removed when prewhitening the "true" signal coming from the star in the time domain.

Some early-type Be stars may exhibit higher stellar frequencies, as the star HD 49330, also observed with CoRoT and analysed in Huat et al. (2009). Variations in frequencies of as high as $16 \mathrm{c} \mathrm{d}^{-1}$ have been detected in this star. For these early-type Be stars, the spectral window produces a more random set of peaks and some caution is required when identifying frequencies coming from the star.

A linear interpolation filling the gaps is also provided by the CoRoT pipeline for the stars observed in the seismology fields. The spectral window of the interpolated data, hence, exhibits peaks neither in the orbital frequency nor day/night frequencies or combinations (see Fig. 3), since there are no gaps. The interpolation does not affect the scientific results for the majority of Be stars since the detected peaks appear at frequencies lower than the orbital frequency. An interpolation of the gaps with 2-order and 3-order polynomial functions give similar results within the errors. Therefore, the interpolation of the gaps does not affect the analysis of the majority of Be stars, although it might affect the analysis of other stars that have higher frequencies. 


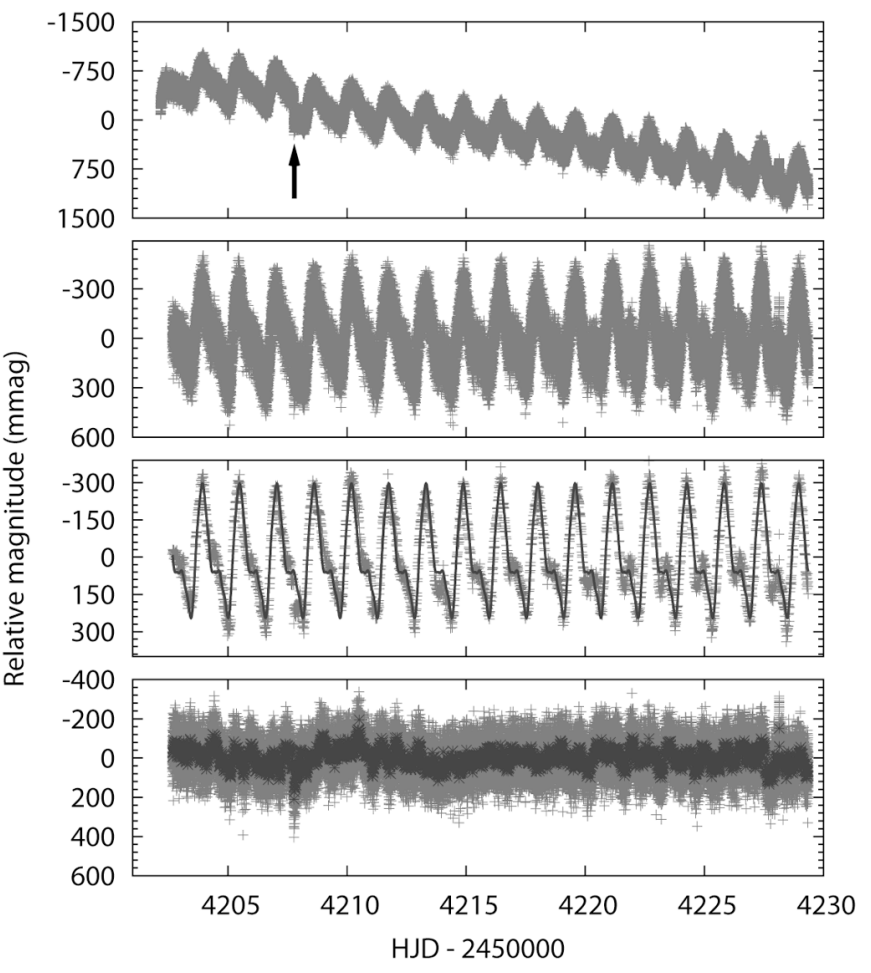

Fig. 4. Top: CoRoT light curve of the star HD 175869. An arrow indicates the time of the jump. Middle top: detrended and corrected from the jump. Middle bottom: blue solid line indicate the fitting of the frequency $F_{1}=0.639 \mathrm{c} \mathrm{d}^{-1}$ and its harmonics. A binning of the light curve was performed to ascertain the match to the fit. Bottom: residuals of the original detrended data (in red) and the binned data (in blue) after prewhitening $F_{1}$ and its harmonics.

\subsubsection{Modulation}

Another instrumental feature is the modulation of the signal with the orbital frequency. The reason is that the CCD response depends on the temperature of the CCD, which depends on the position of the satellite with respect to the Sun, i.e., it varies with the orbital frequency. The signal coming from the star is then multiplied by the signal that varies with the orbit leading to a convolution of two signals in the Fourier domain. In Fig. 3, we see that the orbital frequency $\left(13.97 \mathrm{c} \mathrm{d}^{-1}\right)$ and its harmonics are still present in the periodogram, even after filling the gaps with a linear interpolation.

\subsubsection{Long-term trend}

The CoRoT light curve of HD 175869 (top panel of Fig. 4) shows a long-term trend that is longer than the 27.3 days of observations. This is the combination of two variations, one intrinsic to the star and another related to the instrument. Be stars are expected to have variability of the order of months to years because of changes in the disk (Hubert \& Floquet 1998). In addition, a slope has been detected in the light curve of all stars observed in the seismology fields of CoRoT, probably due to the aging of the CCDs. We remove this long-term trend with a 2nd-degree polynomial function. Nevertheless, some low frequencies close to 0 still appear in the analysis of the light curve. Some of them may be related to the correction of this slope and should be interpreted with caution. Some others can be identified with the beating frequency of the two stellar frequencies with the highest amplitude or modes with $m=0$ (see e.g. Diago et al. 2009). Periods that have not been observed during at least 6 cycles $(P \geq T / 6)$ are considered with caution.

\section{Analysis of the CoRoT light curve of HD 175869}

We present a complete analysis of the CoRoT light curve of the star HD 175869. The long-term trend, clearly seen in the top panel of Fig. 4, is first removed. The change in the temperature of the CCD, which increased slowly at the beginning of the run and then stabilized to a constant value, caused the flux at the beginning to increase slowly until it reached the average flux. For this purpose, we rejected the first 0.5 days of the light curve.

In addition, there is a sudden change in the magnitude at the heliocentric Julian day 2454207.7638 (see arrow in the top panel of Fig. 4). This feature is not of stellar origin, since the discontinuity covers only $32 \mathrm{~s}$. However, at the moment of the jump, there were changes in neither the temperature, nor the pointing of the satellite, and no hot pixel was detected in the background window.

If we divide the data into sets before and after the jump, the two subsets have a duration of 5.1 and 21.6 days respectively. To analyze the full light curve and achieve a higher frequency resolution, we remove a 2 nd-order polynomial fit to each part of the light curve and then merge them into a single dataset. By fitting these polynomial functions to the subsets, we remove periods only as long as the time duration of each subset, i.e., longer than 5 days. However, this procedure is not perfect and the effect of the jump is still visible, adding some extra power.

We also analyzed the complete light curve without correcting for the jump, obtaining a similar set of frequencies mixed with spurious short frequencies.

To perform the frequency analysis, the methods explained in Sect. 2 are applied to the detrended and corrected CoRoT light curve (second panel of Fig. 4), which shows variations of an amplitude of $300 \mu \mathrm{mag}$ and a standard deviation of $180 \mu \mathrm{mag}$. A peak at the frequency $F_{1}=0.639 \mathrm{c} \mathrm{d}^{-1}$ is clearly evident in the periodogram together with its first harmonic $2 F_{1}=1.278 \mathrm{c} \mathrm{d}^{-1}$. Most of the variations come from these two frequencies, which have amplitudes of $\sim 200$ and $\sim 100 \mu \mathrm{mag}$. A more detailed analysis shows that 5 more harmonics with lower amplitudes are detected by all methods.

A comparison of the frequencies detected with Clean_NG, pasper, TiSAFT, and Cleanest was performed. The differences between the frequencies are less than the frequency resolution $\left(0.03745 \mathrm{c} \mathrm{d}^{-1}\right)$ in most cases, as is evident in Fig. 6. Only 3 frequencies differ by little more than the frequency resolution and have small amplitudes $\left(F_{7}=1.945 \mathrm{c} \mathrm{d}^{-1}\right.$ with $10 \mu \mathrm{mag}$, $F_{10}=0.345 \mathrm{c} \mathrm{d}^{-1}$ with $9 \mu \mathrm{mag}$, and $F_{16}=0.769 \mathrm{c} \mathrm{d}^{-1}$ with $7 \mu \mathrm{mag})$. We note that all methods infer similar values for the frequencies with the largest amplitude (for $F_{1}$, the largest difference is $0.0036 \mathrm{c} \mathrm{d}^{-1}$; for $2 F_{1}$ the largest difference is $0.002 \mathrm{c} \mathrm{d}^{-1}$ ).

We then performed a fit of both $F_{1}$ and its 6 harmonics to the light curve by applying a modified version of the Kurtz_bos method, which explores around a given frequency and its 6 first exact harmonics when searching for the minimum variance. The same procedure was also performed with Period04, obtaining similar values. The final fit is superimposed on the light curve in blue in the third panel of Fig. 4. The frequencies, amplitude, and phases are reported in Table 1 . We note that the amplitudes of the frequencies $3 F_{1}$ to $7 F_{1}$ are very low in comparison with those of $F_{1}$ and $2 F_{1}$. The standard deviation of the residuals is $81 \mu$ mag, i.e., $80 \%$ of the variance has been removed from the signal. 
Table 1. Detected frequencies (in $\mathrm{c} \mathrm{d}^{-1}$ and $\mu \mathrm{Hz}$ ), amplitude, and phases found in the analysis of the detrended and corrected light curve.

\begin{tabular}{ccccccc}
\hline \hline$\#$ & $\begin{array}{c}\text { Freq. } \\
\mathrm{c} \mathrm{d}^{-1}\end{array}$ & $\begin{array}{c}\text { Freq. } \\
\mu \mathrm{Hz}\end{array}$ & $\begin{array}{c}\text { Ampl } \\
\mu \mathrm{mag}\end{array}$ & $E_{\text {Ampl }}$ & $\begin{array}{c}\text { Phase } \\
\mathrm{rad} / 2 \pi\end{array}$ & $E_{\text {Phase }}$ \\
\hline$F_{1}$ & 0.639 & 7.3965 & 196.9 & 0.4 & 0.195 & 0.0003 \\
$2 F_{1}$ & 1.278 & 14.7929 & 111.3 & 0.4 & 0.785 & 0.0006 \\
$3 F_{1}$ & 1.917 & 22.1894 & 7.2 & 0.4 & 0.562 & 0.009 \\
$4 F_{1}$ & 2.556 & 29.5858 & 9.7 & 0.4 & 0.342 & 0.007 \\
$5 F_{1}$ & 3.195 & 36.9823 & 8.0 & 0.4 & 0.133 & 0.009 \\
$6 F_{1}$ & 3.834 & 44.3787 & 7.2 & 0.4 & 0.830 & 0.010 \\
$7 F_{1}$ & 4.473 & 51.7752 & 3.7 & 0.4 & 0.035 & 0.018 \\
\hline
\end{tabular}

Here we show only the largest-amplitude frequency $F_{1}$ and its harmonics. The phase was calculated using the time reference HJD $=2454200$. The errors in the amplitude and phase (in Cols. 5 and 7) are calculated for the uncorrelated noise and have to be multiplied by $\sqrt{3}$ to take into account the correlation in the residuals.

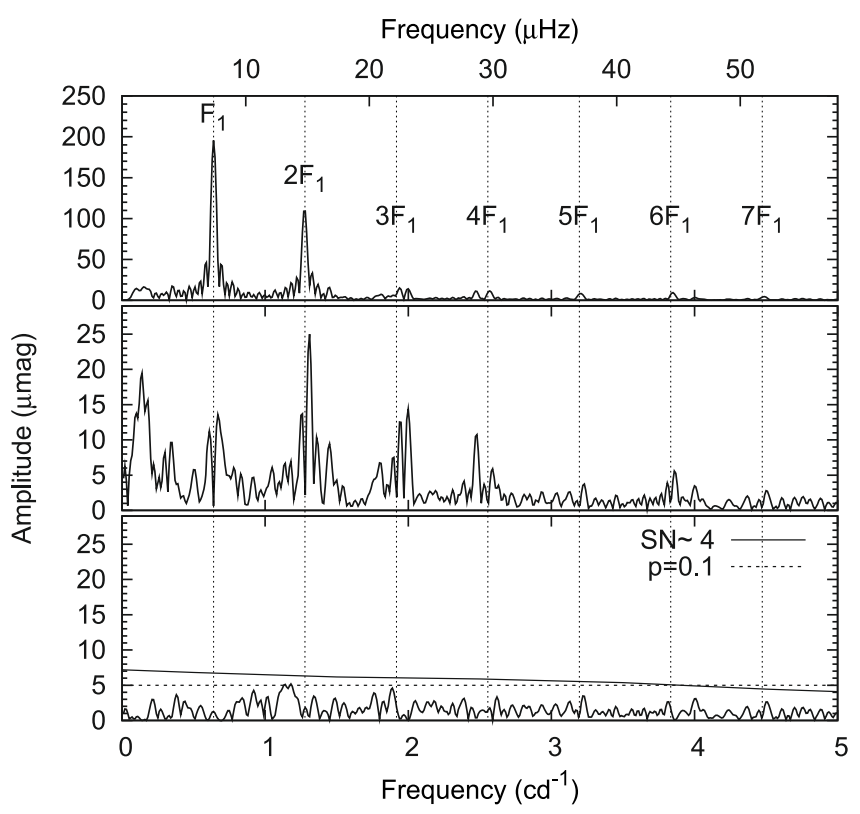

Fig. 5. Periodogram of the: (top) CoRoT light curve; (middle) residuals after prewhitening $F_{1}=0.639 \mathrm{c} \mathrm{d}^{-1}$ and its harmonics; and (bottom) residuals after prewhitening all the significant frequencies. Note the different scale in the first panel with respect to the second and third one. The vertical lines mark the frequency $F_{1}$ and its harmonics in the periodogram. The horizontal lines represent the amplitude threshold for the two stop criteria: the solid line represents the $4 \sigma$ level after the final prewhitening given by pasper, and the dashed line, the threshold for a probability of 0.1 , according to TiSAFT.

In the bottom panel of Fig. 4, we show the residuals of the original data (in red) and the binned data (in blue) after prewhitening $F_{1}$ and its harmonics. The phase diagram with the frequency $F_{1}$, which is displayed in the top panel of Fig. 8, clearly shows a double-wave, with different maxima and minima. This double-wave shape is also seen in the light curve.

We see in the third panel of Fig. 4 that the maxima and minima of the sinusoidal signal are modulated by a long-term trend and that there are some other low-amplitude variations. The periodogram of the residuals after prewhitening $F_{1}$ and its harmonics (middle panel of Fig. 5) shows that the amplitudes of the remaining frequencies are very low $(\leqslant 30 \mu \mathrm{mag})$ but still significant. A close inspection of the periodogram indicates that most of the signal originates in frequencies close to $F_{1}$ and its harmonics. Another set of low frequencies appears around $0.14 \mathrm{c} \mathrm{d}^{-1}$.

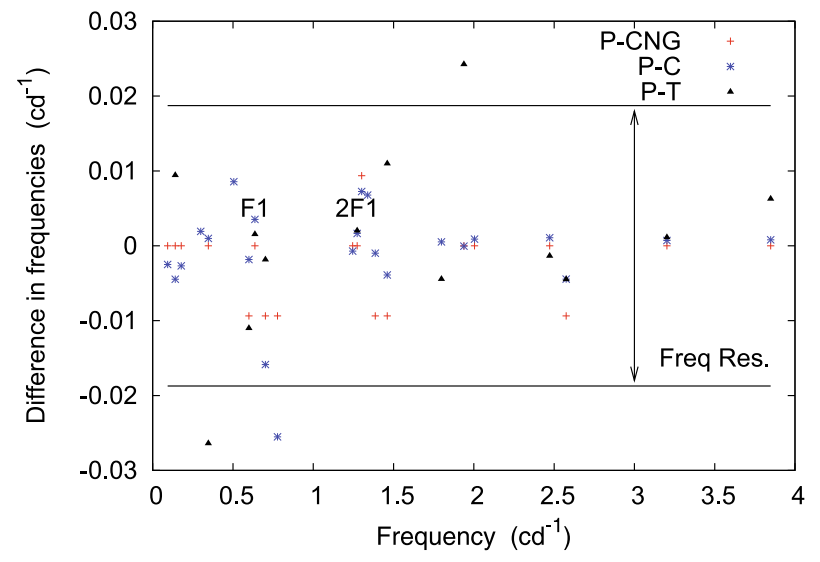

Fig. 6. Differences in frequencies given by the different methods applied to the detrended and corrected CoRoT light curve. Red crosses, blue asterisks, and black triangles represent the differences for pasper and Clean_NG, pasper and Cleanest, and pasper and TiSAFT, respectively.

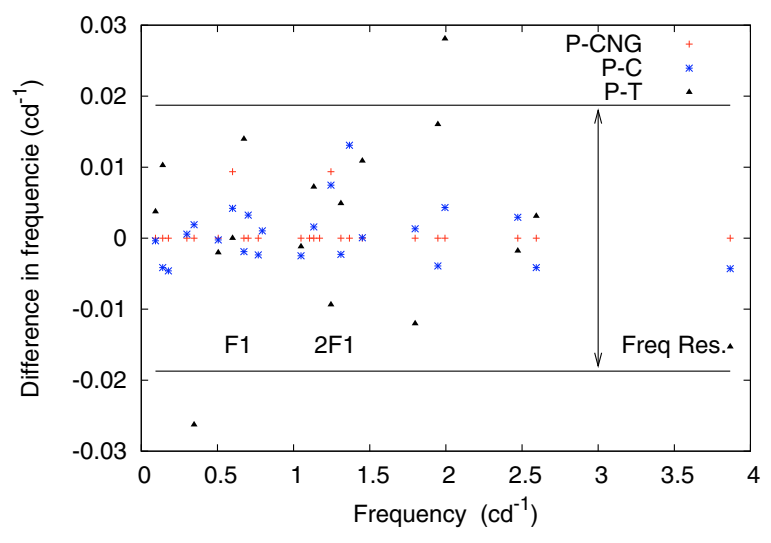

Fig. 7. Differences in frequencies given by the different methods applied to the residuals after prewhitening $F_{1}$ and its harmonics. Red crosses, blue asterisks and black triangles represent the differences for pasper and Clean_NG, pasper and Cleanest, and pasper and TiSAFT, respectively.

A frequency at $F_{2}=1.306 \mathrm{c} \mathrm{d}^{-1}$ is seen in the light curve and detected in the periodogram (middle panel of Fig. 5) with an amplitude of $25 \mu \mathrm{mag}$. The phase diagram, showing the very lowamplitude variation, is depicted in the bottom panel of Fig. 8. We note that the maximum in phase of $F_{2}$ is shifted with respect to the maximum in phase of $F_{1}$ by about 0.25 .

To perform the frequency analysis of the residuals after prewhitening $F_{1}$ and its harmonics, we applied the methods explained in Sect. 2. Again, Clean-NG, pasper, TiSAFT, and Cleanest detected similar frequencies within the frequency resolution, except for two cases, as shown in the Fig. 7.

The non-linear least squares fitting methods (Kurtz_bos, Period04 and Improvef) were then applied to refine the frequencies and obtain the best fit. The final frequencies, amplitude, phases, and signal-to-noise ratio are reported in Table 2. These frequencies are significant, according to Breger et al. (1993), since they have a SNR higher than 4 (see the solid horizontal line in the bottom panel of Fig. 5). In addition, following the statistical test of significance calculated by TiSAFT, the probability that these frequencies are not produced by noise is $90 \%$ (see dashed horizontal line in the figure). Similar frequencies within our frequency resolution have also been found in the second subset, which lasts 21.6 days. 
Table 2. Detected frequencies (in $\mathrm{c} \mathrm{d}^{-1}$ and $\mu \mathrm{Hz}$ ), amplitude, and phases found in the residuals after prewhitening $F_{1}$ and its harmonics.

\begin{tabular}{ccccccccc}
\hline \hline$\#$ & $\begin{array}{c}\text { Freq. } \\
\mathrm{c} \mathrm{d}^{-1}\end{array}$ & $\begin{array}{c}\text { Freq. } \\
\mu \mathrm{Hz}\end{array}$ & $\begin{array}{c}\text { Ampl } \\
\mu \mathrm{mag}\end{array}$ & $\begin{array}{c}E_{\text {Ampl }} \\
\mu \mathrm{mag}\end{array}$ & $\begin{array}{c}\text { Phase } \\
\mathrm{rad} / 2 \pi\end{array}$ & $\begin{array}{c}E_{\text {Phase }} \\
\text { SNR }\end{array}$ & $\begin{array}{c}\text { Comments } \\
\leq 1 / T\end{array}$ \\
\hline$F_{2}$ & 1.306 & 15.114 & 22.2 & 0.4 & 0.959 & 0.003 & 15.1 & $2 F_{1}$ \\
$F_{L 1}$ & 0.141 & 1.634 & 19.8 & 0.4 & 0.196 & 0.003 & 13.5 & \\
$F_{L 2}$ & 0.170 & 1.971 & 19.8 & 0.4 & 0.076 & 0.003 & 13.5 & \\
$F_{3}$ & 2.000 & 23.152 & 12.5 & 0.4 & 0.786 & 0.005 & 8.5 & \\
$F_{L 3}$ & 0.085 & 0.989 & 15.0 & 0.4 & 0.920 & 0.004 & 10.2 & \\
$F_{4}$ & 0.684 & 7.912 & 21.73 & 0.6 & 0.596 & 0.005 & 14.3 & $F_{3}-F_{2}$ \\
$F_{5}$ & 2.475 & 28.644 & 10.68 & 0.4 & 0.817 & 0.006 & 7.3 & \\
$F_{6}$ & 0.601 & 6.959 & 9.79 & 0.4 & 0.349 & 0.006 & 6.7 & $F_{2}-F_{4}$ \\
$F_{7}$ & 1.945 & 22.51 & 10.05 & 0.4 & 0.258 & 0.006 & 6.8 & $3 F_{1}$ \\
$F_{8}$ & 1.256 & 14.541 & 10.46 & 0.4 & 0.314 & 0.006 & 7.1 & $2 F_{1}$ \\
$F_{9}$ & 1.451 & 16.788 & 9.59 & 0.4 & 0.974 & 0.006 & 6.5 & \\
$F_{10}$ & 0.345 & 3.989 & 9.21 & 0.4 & 0.481 & 0.007 & 6.3 & $1 / 2 F_{1}$ \\
$F_{11}$ & 0.699 & 8.085 & 16.67 & 0.6 & 0.757 & 0.006 & 10.9 & $F_{4}$ \\
$F_{12}$ & 1.799 & 20.819 & 7.53 & 0.4 & 0.181 & 0.008 & 5.1 & $F_{5}-F_{4}$ \\
$F_{13}$ & 0.305 & 3.527 & 6.80 & 0.4 & 0.776 & 0.009 & 4.6 & $1 / 2 F_{1}$ \\
$F_{14}$ & 1.383 & 16.001 & 7.48 & 0.4 & 0.248 & 0.008 & 5.1 & $2 F_{4}$ \\
$F_{15}$ & 0.507 & 5.868 & 6.65 & 0.4 & 0.174 & 0.009 & 4.5 & $F_{5}-F_{4}-F_{1}$ \\
$F_{16}$ & 0.769 & 8.898 & 7.20 & 0.4 & 0.683 & 0.009 & 4.9 & $F_{9}-F_{4}$ \\
$F_{17}$ & 1.048 & 12.126 & 6.29 & 0.4 & 0.0269 & 0.01 & 4.3 & $F_{10}+F_{11}$ \\
$F_{18}$ & 2.590 & 29.978 & 5.89 & 0.4 & 0.758 & 0.01 & 4.0 & $4 F_{1}$ \\
$F_{19}$ & 3.863 & 44.714 & 5.77 & 0.4 & 0.430 & 0.01 & 3.9 & $6 F_{1}$ \\
\hline
\end{tabular}

The phase was calculated using the time reference HJD $=2454200.0$. Idem as Table 1 for the errors. The signal-to-noise ratio obtained with the program pasper is given in Col. 8. The last column indicates the interpretation for each frequency as independent, harmonic, or combination, following the present frequency resolution, which is $1 / T=0.037 \mathrm{c} \mathrm{d}^{-1}$. Long-term frequencies are marked with $F_{\mathrm{L}}$. For details, see text.

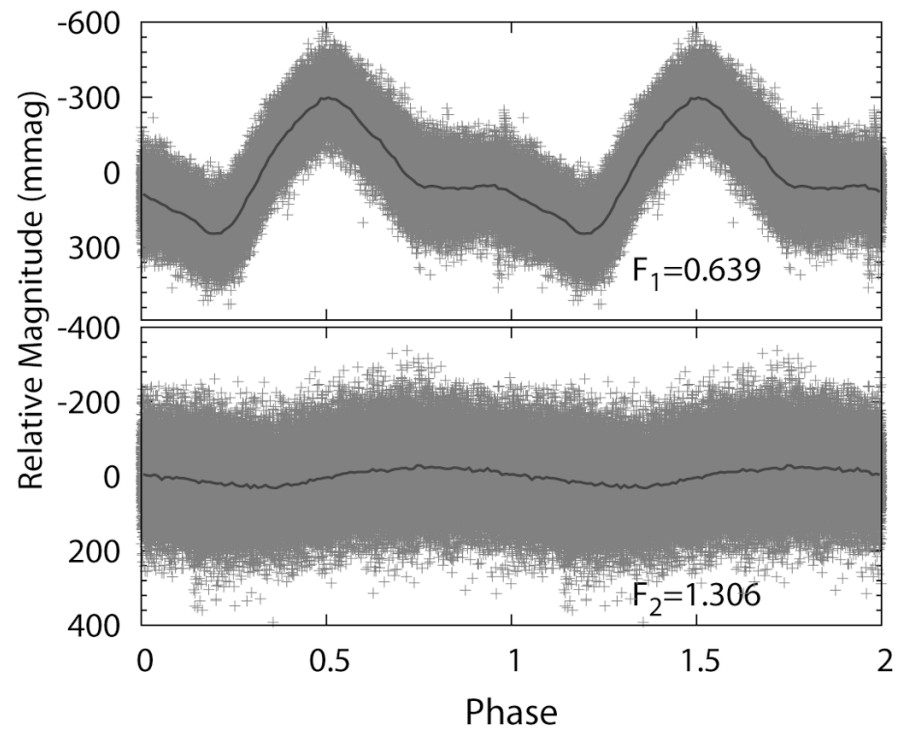

Fig. 8. Top: phase diagram of the light curve folded with the frequency $F_{1}$. Note the unequal maxima and minima. Bottom: phase diagram of the residuals after prewhitening $F_{1}$ and its harmonics folded with the frequency $F_{2}=1.306 \mathrm{c} \mathrm{d}^{-1}$. The solid blue line represents the binned data.

It is remarkable that most of the new frequencies appear close to $F_{1}$ and its harmonics (see vertical lines in Fig. 5). The amplitude of these frequencies is at least 10 times lower than the amplitude of the main frequency $F_{1}$. However, some frequencies (see Table 2) differ from $F_{1}$ and its harmonics and their combinations at the present frequency resolution, and could thus be interpreted as independent frequencies.

A change in the amplitude of the frequency $F_{1}$ and its harmonics would also produce several peaks around these frequencies. By cutting the light curve into several pieces and calculating the amplitudes of the two main frequencies $F_{1}$ and $2 F_{1}$, we see a clear variation during the course of the 27 days (see Fig. 9). The frequency $F_{1}$ has the largest amplitude around the time 2454210 , just when $2 F_{1}$ reaches its minimum. The plot shows that, at least at the beginning of the run, the two frequencies exchange their values of power, i.e., the power of $F_{1}$ increases when the power of $F_{2}$ decreases, and vice versa. Unfortunately, the short duration of the light curve does not allow us to perform a time-frequency analysis, as applied in the study of other Be stars observed during a longer run (see e.g., Diago et al. 2009).

In addition, long periods of around 12,7 , and 6 days $(0.08$, $0.14,0.17 \mathrm{c} \mathrm{d}^{-1}$; marked in Table 2 with $F_{\mathrm{L}}$ ) appear in the periodogram. However, we cannot confirm that these frequencies are of stellar origin and they must be interpreted with caution. They are probably related to long-term trends, which Fourier methods should detect as a frequency and its harmonics. However, these 3 long periods also appear in the analysis of the second subset of the light curve, which does not exhibit any instrumental jump.

\section{Spectroscopic analysis}

The fundamental parameters of HD 175869 were determined from high-resolution spectra obtained during the preparation of the CoRoT mission and collected in the GAUDI database by Frémat et al. (2006). These parameters, which were corrected for gravitational darkening effects (Frémat et al. 2005), are reported in Table 3. Using the evolutionary tracks taken from Schaller et al. (1992), we derive the equatorial radius and the rotational frequency (see the Table), as well as the mass $M=4.3 \pm 0.2 M_{\odot}$ and luminosity $\log L=2.86 \pm 0.11 L_{\odot}$.

New spectroscopic observations were acquired between June 25 and July 6, 2007 at the David Dunlap Observatory (DDO) in Toronto with the $1.88 \mathrm{~m}$ telescope. A total of $100 \mathrm{spec}-$ tra were gathered for the spectral range 4300-4600 $\AA$ with a resolving power of 10000 , a exposure time of between 600 to 


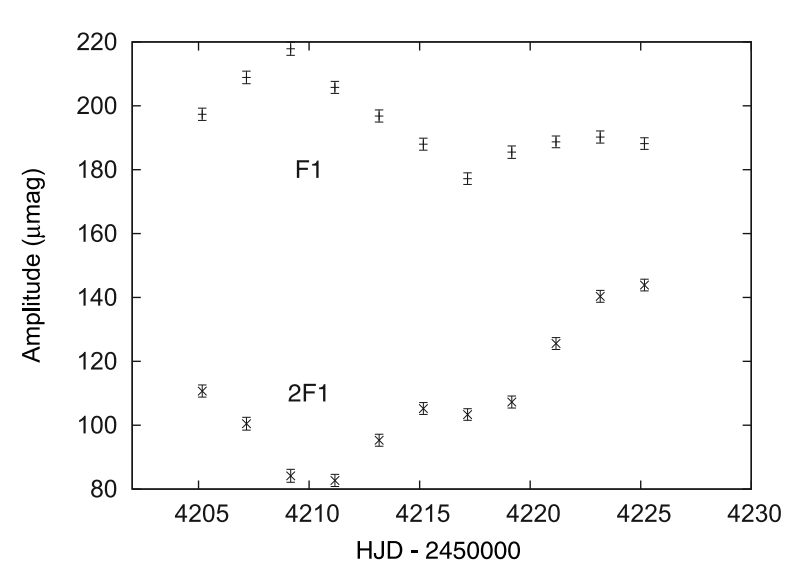

Fig. 9. Amplitude variations in the main frequency and its first harmonic.

Table 3. Stellar parameters $\left(i, T_{\text {eff }}, \log g, V \sin i\right)$, corrected for the veiling and the effects of the fast rotation, the derived equatorial radius, and rotational velocity of HD 175869 for different values of $\Omega / \Omega_{\mathrm{c}}$, where $\Omega$ and $\Omega_{\mathrm{c}}$ are the angular velocity and its critical value, respectively.

\begin{tabular}{ccccccc}
\hline \hline$\Omega / \Omega_{\mathrm{c}}$ & $\begin{array}{c}i \\
(\mathrm{deg})\end{array}$ & $\begin{array}{c}T_{\text {eff }} \\
(\mathrm{K})\end{array}$ & $\begin{array}{c}\log g \\
(\mathrm{cgs})\end{array}$ & $\begin{array}{c}V \sin i \\
\left(\mathrm{~km} \mathrm{~s}^{-1}\right)\end{array}$ & $\begin{array}{c}R_{\mathrm{eq}} \\
R_{\odot}\end{array}$ & $\begin{array}{c}f_{\text {rot }} \\
\mathrm{c} \mathrm{d}^{-1}\end{array}$ \\
\hline 0.80 & 58 & 12500 & 3.52 & 168 & 6.8 & 0.58 \\
0.90 & 47 & 12000 & 3.47 & 171 & 7.3 & 0.64 \\
0.95 & 44 & 12000 & 3.40 & 180 & 8.6 & 0.60 \\
0.99 & 43 & 12000 & 3.39 & 196 & 9.2 & 0.68 \\
\hline
\end{tabular}

The estimated errors in $T_{\text {eff }}, \log g, V \sin i$, equatorial radius, and rotational frequency are $10 \mathrm{deg}, 600 \mathrm{~K}, 0.12 \mathrm{dex}, 10 \mathrm{~km} \mathrm{~s}^{-1}, 2.4 R_{\odot}$, and $0.1 \mathrm{c} \mathrm{d}^{-1}$, respectively.

$2000 \mathrm{~s}$, and a signal-to-noise ratio of between 150 and 300 . We reduced the spectra with $\operatorname{IRAF}^{1}$ using standard techniques for CCD data. All the data were corrected for heliocentric velocities.

A set of spectropolarimetric observations was also obtained with Narval mounted on the $2 \mathrm{~m}$ Télescope Bernard Lyot (TBL) at Pic du Midi from June 23 to July 4, 2007 (PI: Catala). Eighteen échelle spectra were collected of signal-to-noise ratio of between 300 and 500. The resolution is 65000 and the wavelength ranges from 3690 and $10480 \AA$. They were obtained in spectrolarimetric mode in Stokes $V$ sequences of $4 \times 600$ s each. The reduction of the spectra was performed using the LibreEsprit pipeline (Donati et al. 1997) at the telescope by the TBL team. The spectra were corrected for heliocentric velocities. The $\mathrm{H} \alpha$ and $\mathrm{H} \beta$ profiles for the star HD 175869 are displayed in Fig. 10. An averaging of the 18 Narval spectra was performed.

The fundamental parameters were redetermined with the new spectra obtained with Narval, following the same procedure as Frémat et al. (2006). The first step was to compare observed spectra in the 4000-4500 $\AA$ region to a grid of synthetic spectra by using a least squares method. The grid of stellar fluxes was computed with plane-parallel model atmospheres for different values of the effective temperature and surface gravity.

The model atmospheres that we used for effective temperatures lower than $27000 \mathrm{~K}$ are those calculated by Kurucz (1993) and Castelli \& Kurucz (2003) in LTE. However, for stars hotter than $15000 \mathrm{~K}$ and cooler than $27000 \mathrm{~K}$, non-LTE level

\footnotetext{
${ }^{1}$ IRAF is distributed by the National Optical Astronomy Observatories, which is operated by the Association of Universities for Research in Astronomy (AURA) Inc., under cooperative agreement with the National Science Foundation.
}
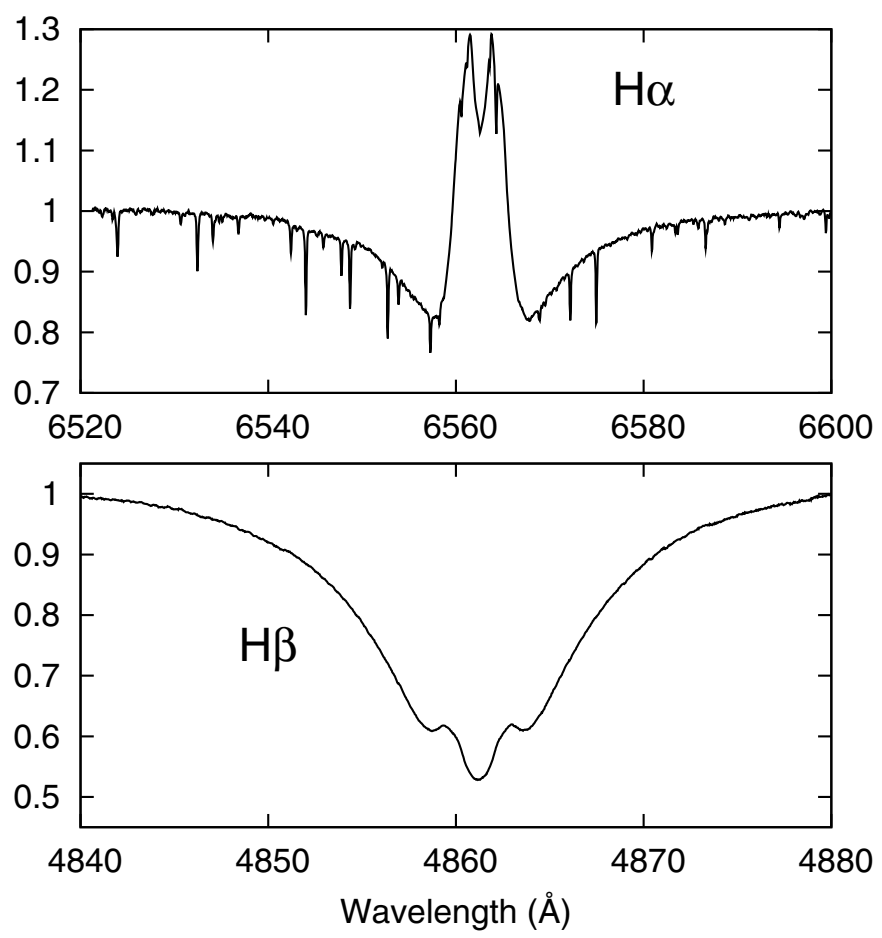

Fig. 10. The averaged $\mathrm{H} \alpha$ and $\mathrm{H} \beta$ profiles of the star HD 175869.

populations were calculated for each of the considered atoms using the TLUSTY code (Hubeny \& Lanz 1995) and keeping the temperature and density distributions fixed. The surface chemical abundances that we adopted are those published by Grevesse \& Sauval (1998) for the Sun. Table 2 of Frémat et al. (2006) lists the ions that we used in the computations.

At this step, we found the following apparent fundamental parameters: $T_{\text {eff }}=11794 \mathrm{~K}, \log g=3.38 \mathrm{dex}$, and $V \sin i=166 \mathrm{~km} \mathrm{~s}^{-1}$. The observed (in red) and the fitted synthetic spectrum (in blue) are shown in Fig. 11 for these parameters. We note that these values are similar within their errors to those in Table 4 of Frémat et al. (2006)

The next step was to correct the veiling caused by the emission from the envelope and to take into account the effects of the stellar flattening and gravitational darkening caused by the rapid rotation. The complete procedure provided us with different parameter values estimated at different $\Omega / \Omega_{\mathrm{c}}$. The parameters determined for the new Narval spectra are totally consistent with those found by Frémat et al. (2006), shown in Table 3.

To confirm the photometric frequencies detected for CoRoT data, we performed a time series analysis of the new set of spectra. Fourier Transform and least squares fitting methods as in Neiner et al. (2005) were applied to each resolution bin of line-profile time series of the He I 4387 and 4471, Mg II 4481, and $\mathrm{H} \gamma$ lines. The line profiles do not show any coherent variation. The frequencies observed in the CoRoT data were also not detected in the spectroscopy data. This is probably because of the low amplitude of the frequencies obtained in the CoRoT light curve.

We applied the least square deconvolution (LSD) technique (Donati et al. 1997) to the 18 spectropolarimetric Stokes $V$ data obtained with Narval to search for the presence of a magnetic field. The LSD method combines the very small circularly polarised signatures, properly weighted, of all available lines in the spectrum to increase the signal-to-noise ratio. For HD 175869 , we used 81 different lines. The mean signal-to-noise ratio of the 

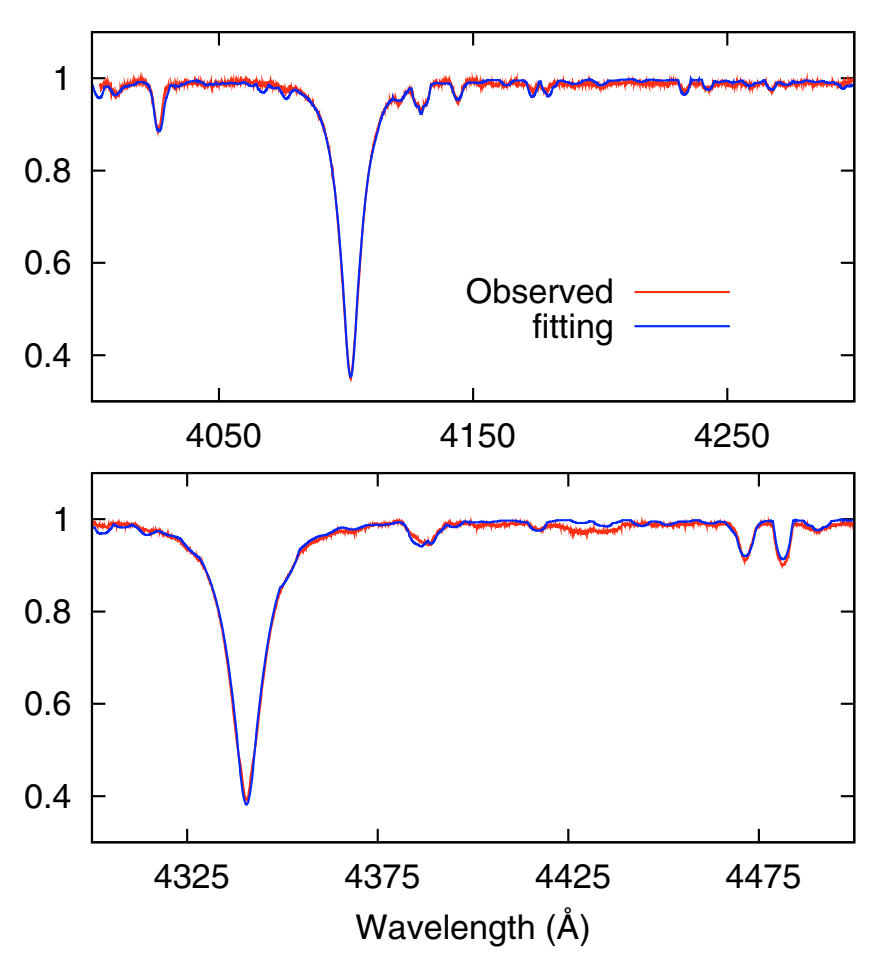

Fig. 11. Comparison between observed (in red) and the best-fit synthetic spectrum (in blue) with the apparent fundamental parameters $T_{\text {eff }}=11794 \mathrm{~K}, \log g=3.38 \mathrm{dex}$ and $V \sin i=166 \mathrm{~km} \mathrm{~s}^{-1}$.

summed Stokes $V$ profile is $\sim 3000$. No evidence of a Zeeman signature is detected in the summed profile. Moreover, all 18 resulting measurements of the longitudinal magnetic field are compatible with 0 . Assuming that the magnetic field, if it exists, is dipolar, and considering the error bars of our measurements, it should have a longitudinal component weaker than $400 \mathrm{G}$. Local stronger magnetic fields could exist if the hypothetical magnetic field was more complex than dipolar. However, for massive stars such as HD 175869, fields known that are often dominated by a dipolar component (see e.g., Neiner et al. 2003a).

\section{Discussion}

The high accuracy of the CoRoT data has allowed us to detect very low-amplitude frequencies in the B8IIIe star HD 175869. The light-curve shape has a double wave, as shown in the third panel of Fig. 4. Two main frequencies are detected at $F_{1}=$ $0.639 \mathrm{c} \mathrm{d}^{-1}$ and its first harmonic $F_{1}=1.278 \mathrm{c} \mathrm{d}^{-1}$ and they contribute to most of the variation ( $79 \%$ of the variance). The 5 next harmonics of $F_{1}$ have also been detected, with much lower amplitude (see Table 1).

In the third panel of Fig. 4, we display the fitting of $F_{1}$ and its harmonics in a blue line superimposed on the light curve. The maxima and minima change slowly with time, suggesting the presence of low-amplitude frequencies. A total of 21 significant frequencies with amplitudes decreasing from 23 to few $\mu$ mag have been detected in the residuals after prewhitening $F_{1}$ and its harmonics. A peak at the frequency $F_{2}=1.306 \mathrm{c} \mathrm{d}^{-1}$ clearly appears in the periodogram, displayed in the middle panel of Fig. 5. As depicted in the same figure, most of the detected frequencies are close to $F_{1}$ and its harmonics. Among these 21 detected frequencies, three are low frequencies, which are interpreted with caution, and only 3 frequencies $\left(F_{3}=2.000, F_{5}=2.475\right.$ and $\left.F_{9}=1.451 \mathrm{c} \mathrm{d}^{-1}\right)$ are independent of our frequency resolution $\left(1 / T=0.037 \mathrm{c} \mathrm{d}^{-1}\right)$. The presence of these low-amplitude frequencies can be interpreted as amplitude or phase variations in the main frequencies. We have indeed shown in Fig. 9 that $F_{1}$ and $2 F_{1}$ exhibit amplitude variations of the order of $20 \mu \mathrm{mag}$.

The physical parameters have been calculated from highresolution spectra, taking into account the rapid rotation of the Be star (see Table 3). New high-resolution spectra have been obtained and a new determination of the parameters has been performed, obtaining similar values. A rotational frequency of $f_{\text {rot }}=0.63 \pm 0.2 \mathrm{c} \mathrm{d}^{-1}$ has been derived. A time series analysis of the spectroscopic data has been performed, which yielded negative results. We have also not found evidence of a magnetic field in this star, for an upper limit of $B_{\text {long }}=400 \mathrm{G}$.

Since the main frequency $F_{1}=0.639 \mathrm{c} \mathrm{d}^{-1}$ is compatible with the rotational frequency, and the phase diagram exhibits a double wave (upper panel of Fig. 8), the observed variation detected in this late-type Be star should be able to be interpreted as the signature of rotational modulation (Balona 1990). For this interpretation, the varying relative size of photospheric signatures (either spots or clouds) in or close to the photosphere should produce the change in amplitude observed in the light curve. Another Be star, Omi And, also shows variations, which were interpreted by Sareyan et al. (1998) as stellar activity. Moreover, Omi And also presents amplitude variations over a few days.

Unfortunately, in the analysis of the spectropolarimetric data, no sign of magnetic cloud, starspot, or activity has been detected. A magnetic field could fix the inhomogeneities to or above the photosphere and thus explain why we observe the same period of variations throughout the entire run.

An alternative explanation is that the variations detected in the star HD 175869 could be due to non-radial pulsations. Saio et al. (2007) detected a few significant frequencies in the MOST data of the late-type $\mathrm{Be} \operatorname{star} \beta \mathrm{CMi}$, and interpreted them as prograde g-modes. The same interpretation could be applied to the frequencies detected in HD 175869. The identification of the pulsation modes will require models that take into account the characteristics of Be stars, in particular rapid rotation. The amplitude variations could also be explained by the beating of multiple close pulsation modes, which are not detected because of the short time-span of the run and the corresponding low frequency resolution.

To verify whether the hypothesis of non-radial pulsations in the star HD 175869 is plausible, we have placed its parameters in the HR diagram and compared with the instability strips of SPB stars (see Fig. 12). The instability strips are taken from Miglio et al. (2007), for an overshooting of $0.2 \mathrm{Hp}$ with OPAL (solid red line) and OP (dashed green line) opacities, and from Pamyatnykh (1999), with OP opacities and no overshooting (dotted blue line). In both cases, chemical mixtures are taken from Asplund et al. (2005).

Following Pamyatnykh (1999), the low temperature boundary of the instability strip of SPB stars coincides with the TAMS because of the strong damping of the higher-order gravity modes in the interiors of post-MS stellar models. As shown in Fig. 12, the star is far away from the strip calculated without overshooting (dotted blue line). This would suggest that HD 175869 is unable to exhibit non-radial pulsations and therefore the observed variations should be due to rotational modulation.

However, the inclusion of the overshooting from the stellar convective cores in the models causes the MS-stage duration to increase because of the enlargement of the mixed core. The main effect of the overshooting in the HR diagram is to extend the 


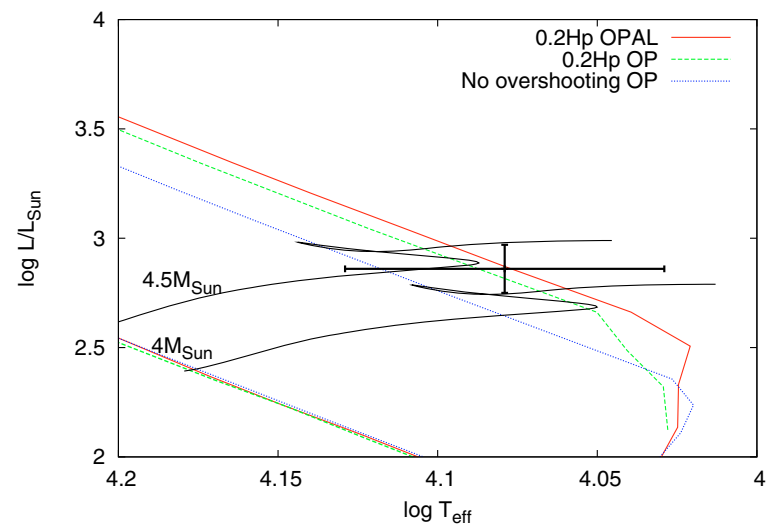

Fig. 12. Position of the Be star HD 175869 in the HR diagram. The dotted blue line represents the instability strip for SPB stars without overshooting computed by Pamyatnykh (1999), while the solid red and dashed green lines indicate the strip for an overshooting of $0.2 \mathrm{Hp}$ with OPAL and OP opacities, respectively, taken from Miglio et al. (2007). Two evolutionary tracks for 4.5 and $4 M_{\odot}$ are also depicted.

MS band by a shift in the TAMS line (see solid red and dashed green lines in Fig. 12). The same results are produced by rotational mixing, which brings $\mathrm{H}$-fuel into the convective core. The displacement of the TAMS because of rotation mimics the overshooting effect. Since HD 175869 is a rapidly-rotating star, we expect rotational mixing and therefore, the pulsation hypothesis is also possible. The marginal position of HD 175869 in the instability strip of SPBs would explain the low-amplitude variations detected in the CoRoT light curve.

\section{Conclusions}

The origin of the short-term variability observed in Be stars has been an open question for a long time. Most Be stars exhibit variations that can be modeled as non-radial pulsations (Rivinius et al. 2003). However, other Be stars show particular variations that cannot be modeled as non-radial pulsations (see Porter \& Rivinius 2003). At least one Be star, namely $\omega$ Ori exhibits variations caused by, at the same time, pulsations and inhomogeneities rotating with the star (Neiner et al. 2003b). The most likely interpretation of the variations in the CoRoT light curve of the Be star HD 175869 is the presence of stellar activity attached to the star, although a pulsational interpretation cannot be completely excluded. Unfortunately, no magnetic field has been detected in the spectropolarimetric data.

Pulsations are commonly found in late-type Be stars inside the SPB instability strip (Pamyatnykh 1999), indicating that they are likely to be related gravity modes. Although the instability strip has not been calculated for such rapid rotating stars, the fact that HD 175869 is significantly far away from the strip supports the hypothesis that the variations detected are due to rotational modulation. However, the rotational mixing of the chemical abundances in the interior may have extended the duration of the star on the MS and thus the star may still be burning hydrogen in its core. Therefore, the pulsation hypothesis is also plausible.

Acknowledgements. The CoRoT space mission, launched on December 27th 2006, has been developed and is operated by CNES, with the contribution of
Austria, Belgium, Brasil, ESA, Germany and Spain. This research is also based on data obtained at the David Dunlap Observatory (University of Toronto) and at the Télescope Bernard Lyot (Pic du Midi). We are grateful to D. H. Roberts for sending us his version of the CLEAN algorithm, and to D. Potts, S. Kunis, and J. Keiner for making their software available to the scientific community. We also thank Andrea Miglio for being so kind to send us the instability strips of B stars. We acknowledge the anonymous referee for the useful comments. The research of J.F. and J.S. has been financed by the Spanish Plan Nacional de Investigación Científica, Desarrollo e Innovación Tecnológica, and FEDER, through the contract AYA2007-62487. The work of P.D.D is supported by a FPU grant from the Spanish "Ministerio de Educación y Ciencia”. R.G. acknowledges the financial support from the program ESP2007-65480-C02-01.

\section{References}

Asplund, M., Grevesse, N., Sauval, A. J., Allende Prieto, C., \& Blomme, R. 2005, A\&A, 431, 693

Auvergne, M., Bodin, P., Boisnard, L., et al. 2009, A\&A, 506, 411

Balona, L. A. 1990, MNRAS, 245, 92

Balona, L. A. 1995, MNRAS, 277, 1547

Breger, M., Stich, J., Garrido, R., et al. 1993, A\&A, 271, 482

Castelli, F., \& Kurucz, R. L. 2003, ed. N. Piskunov, W. W. Weiss, \& D. F. Gray, IAU Symp., 20

Deeming, T. J. 1975, Ap\&SS, 36, 137

Diago, P. D., Gutiérrez-Soto, J., Fabregat, J., \& Martayan, C. 2008, A\&A, 480, 179

Diago, P. D., Gutiérrez-Soto, J., Auvergne, M., et al. 2009, A\&A, 506, 125

Donati, J.-F., Semel, M., Carter, B. D., Rees, D. E., \& Collier Cameron, A. 1997, MNRAS, 291, 658

Fisher, R. A. 1929, Proc. Roy. Soc. London Ser., 125, 54

Foster, G. 1995, AJ, 109, 1889

Frémat, Y., Zorec, J., Hubert, A.-M., \& Floquet, M. 2005, A\&A, 440, 305

Frémat, Y., Neiner, C., Hubert, A.-M., et al. 2006, A\&A, 451, 1053

Galassi, M., Davies, J., Theiler, J., et al. 2006, GNU Scientific Library Reference Manual, revised and updated second edition, GNU Free Software Foundation, The corresponding software can be found at http: //www.gnu.org/software/gsl/

Grevesse, N., \& Sauval, A. J. 1998, Space Sci. Rev., 85, 161

Högbom, J. A. 1974, A\&AS, 15, 417

Huat, A.-L., Leroy, B., \& Diago, P.-D. 2008, in SF2A-2008: Proceedings of the Annual meeting of the French Society of Astronomy and Astrophysics, ed. C. Charbonnel, F. Combes, \& R. Samadi, http://proc.sf2a.asso.fr

Huat, A.-L., Hubert, A.-M., Baudin, F., et al. 2009, A\&A, 506, 95

Hubeny, I., \& Lanz, T. 1995, ApJ, 439, 875

Hubert, A. M., \& Floquet, M. 1998, A\&A, 335, 565

Kallinger, T., Reegen, P., \& Weiss, W. W. 2008, A\&A, 481, 571

Kurtz, D. W. 1985, MNRAS, 213, 773

Kurucz, R. L. 1993, CD-ROM No. 13 (Cambridge, Mass.: Smithsonian Astrophysical Observatory)

Lenz, P., \& Breger, M. 2005, Commun. Asteroseismol., 146, 53

Miglio, A., Montalbán, J., \& Dupret, M.-A. 2007, Commun. Asteroseismol., 151,48

Montgomery, M., \& O’Donoghue, D. 1999, Delta Scuti Newsletter, 13, 28

Neiner, C., Geers, V. C., Henrichs, H. F., et al. 2003a, A\&A, 406, 1019

Neiner, C., Hubert, A.-M., Frémat, Y., et al. 2003b, A\&A, 409, 275

Neiner, C., Floquet, M., Hubert, A. M., et al. 2005, A\&A, 437, 257

Neiner, C., Gutiérrez-Soto, J., Baudin, F., et al. 2009, A\&A, 506, 143

Pamyatnykh, A. A. 1999, Acta Astron., 49, 119

Perryman, M. A. C., Lindegren, L., Kovalevsky, J., et al. 1997, A\&A, 323, L49 Porter, J. M., \& Rivinius, T. 2003, PASP, 115, 1153

Potts, D., Steidl, G., \& Tasche, M. 2001, in Modern Sampling Theory: Mathematics and Applications, ed. J. J. Benedetto, \& P. Ferreira, Applied and Numerical Harmonic Analysis Series (Boston: Birkhauser), 249, A library that implements in $\mathrm{C}$ the algorithms described here is freely available at http://www-user.tu-chemnitz.de/ potts/nfft/

Powell, M. J. D. 1964, The Computer Journal, 7, 155

Rivinius, T., Baade, D., \& Štefl, S. 2003, A\&A, 411, 229

Roberts, D. H., Lehar, J., \& Dreher, J. W. 1987, AJ, 93, 968

Saio, H., Cameron, C., Kuschnig, R., et al. 2007, ApJ, 654, 544

Sareyan, J. P., Gonzalez-Bedolla, S., Guerrero, G., et al. 1998, A\&A, 332, 155

Schaller, G., Schaerer, D., Meynet, G., \& Maeder, A. 1992, A\&AS, 96, 269

Schwarzenberg-Czerny, A. 1991, MNRAS, 253, 198 